\title{
0081 INTEGRATING IPV AND HIV PREVENTION INTO HOSPITAL SERVICES IN THAILAND
}

SGrisurapong* Correspondence: Faculty of Social Sciences and Humanities, Mahidol University, Salaya, Puthamonthon 4 Road Nakhonpathom 73170, Thailand

\subsection{6/ip.2010.029215.81}

This project aimed to integrating program of intimate partner violence (IPV) and HIV prevention into hospital services by first identification of the interrelation between IPV and HIV which focusing on sexual relationship power in terms of ability to negotiate for safe sex and condom use.

Data had been collected from two women groups: victims of violence (86 cases) and HIV positive (205 cases) and two control groups: general women (314 cases) and pregnant women (300 cases) in a selected province in the East of Thailand.

An interview had been conducted to obtain data on socioeconomic \& demographic characteristics, physical and sexual violence experience, sexual relationship power \& sexual risk behaviour and attitude towards gender role of respondents. The questionnaire of WHOs project on multi-country study of violence against women and Sexual Relationship Power Scale developed by Pulerwitz (2000) had been employed as a guideline for interviews.

The results revealed that being victims of violence was significantly related to less asking partners to use condoms \& being refusal by partners to use, having forced first sexual intercourse, extramarital sex and experience of sexually transmitted disease infection compared to control groups. Less sexual relationship power were the key factors to negotiate for sexual relationship and condom use. 
These findings had been used to develop an IPV screening program in the pilot hospital and a cross-training for hospital staffs dealing with IPV and HIV. This initiative can prevent women victims of violence from HIV infection and alleviate HIV positive women from IPV due to their HIV status. 